\title{
SELF-SIMILAR MEASURES AND THEIR FOURIER TRANSFORMS. II
}

\author{
ROBERT S. STRICHARTZ
}

AbSTRACT. A self-similar measure on $\mathbf{R}^{n}$ was defined by Hutchinson to be a probability measure satisfying

$$
\mu=\sum_{j=1}^{m} a_{j} \mu \circ S_{j}^{-1},
$$

where $S_{j} x=\rho_{j} R_{j} x+b_{j}$ is a contractive similarity $\left(0<\rho_{j}<1, R_{j}\right.$ orthogonal) and the weights $a_{j}$ satisfy $0<a_{j}<1, \sum_{j=1}^{m} a_{j}=1$. By analogy, we define a self-similar distribution by the same identity $(*)$ but allowing the weights $a_{j}$ to be arbitrary complex numbers. We give necessary and sufficient conditions for the existence of a solution to $(*)$ among distributions of compact support, and show that the space of such solutions is always finite dimensional.

If $F$ denotes the Fourier transformation of a self-similar distribution of compact support, let

$$
H(R)=\frac{1}{R^{n-\beta}} \int_{|x| \leq R}|F(x)|^{2} d x,
$$

where $\beta$ is defined by the equation $\sum_{j=1}^{m} \rho_{j}^{-\beta}\left|a_{j}\right|^{2}=1$. If $\rho_{j}^{\nu_{j}}=\rho$ for some fixed $\rho$ and $\nu_{j}$ positive integers we say the $\left\{\rho_{j}\right\}$ are exponentially commensurable. In this case we prove (under some additional hypotheses) that $H(R)$ is asymptotic (in a suitable sense) to a bounded function $\widetilde{H}(R)$ that is bounded away from zero and periodic in the sense that $\widetilde{H}(\rho R)=\widetilde{H}(R)$ for all $R>0$. If the $\left\{\rho_{j}\right\}$ are exponentially incommensurable then $\lim _{R \rightarrow \infty} H(R)$ exists and is nonzero.

\section{INTRODUCTION}

In Part I [S3] we studied Fourier transforms of self-similar measures, such as the usual Cantor measure, showing in some cases that averages such as

$$
H(R)=\frac{1}{R^{n-\beta}} \int_{|x| \leq R}|\hat{\mu}(x)|^{2} d x
$$

remain bounded above and below as $R \rightarrow \infty$, where $n$ is the dimension of the Euclidean space $\mathbf{R}^{n}$ on which $\mu$ is defined and $\beta$ is a kind of dimension determined by the self-similarity properties of $\mu$. But now look at Figure 1, which shows the graph of $H(R)$ on a logarithmic scale for $R$, where $\mu$ is usual Cantor measure, suitably normalized. Clearly $H(R)$ is asymptotically multiplicatively periodic.

Received by the editors July 17, 1990 and, in revised form, December 13, 1990.

1980 Mathematics Subject Classification (1985 Revision). Primary 42B10.

Research supported in part by the National Science Foundation, Grant DMS-8902216. 


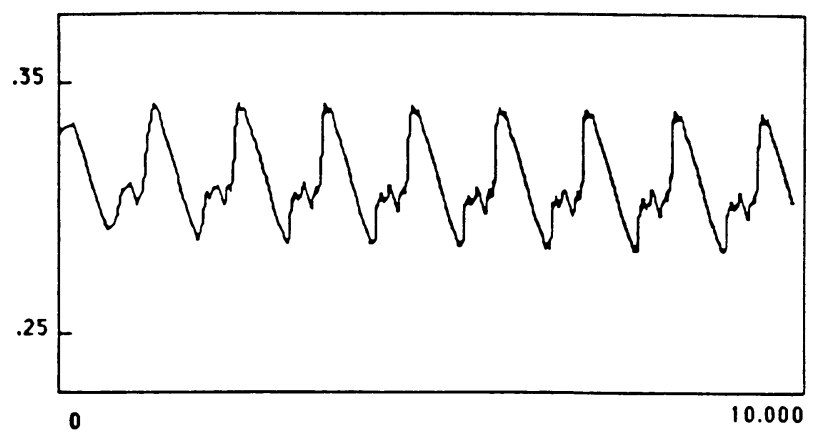

FIGURE 1. The graph of $H(R)$ on a logarithmic $R$ scale for $\hat{\mu}(x)=\prod_{k=1}^{\infty} \cos \pi 3^{-k} x$, corresponding to a normalized Cantor measure.

$$
H(R) \sim \widetilde{H}(R) \quad \text { as } R \rightarrow \infty,
$$

where

$$
\widetilde{H}(\rho R)=\widetilde{H}(R)
$$

( $\rho=\frac{1}{3}$ for the Cantor measure). One goal of this paper is to prove this sort of asymptotic periodicity in a number of cases.

Self-similar measures are defined by Hutchinson $[\mathrm{H}]$ to be probability measures $\mu$ on $\mathbf{R}^{n}$ that satisfy an identity

$$
\mu=\sum_{j=1}^{m} a_{j} \mu \circ S_{j}^{-1}
$$

for a set of positive weights $a_{j}$ satisfying

$$
\sum_{j=1}^{m} a_{j}=1
$$

and a set of contractive similarity transformations $S_{j}$ of $\mathbf{R}^{n}$. Here we write $\mu \circ S_{j}^{-1}$ for the measure satisfying

$$
\int \varphi d \mu \circ S_{j}^{-1}=\int \varphi \circ S_{j} d \mu
$$

and $S_{j} x=\rho_{j} R_{j} x+b_{j}$, where $0<\rho_{j}<1, R_{j}$ is an orthogonal transformation on $\mathbf{R}^{n}$, and $b_{j}$ is a vector in $\mathbf{R}^{n}$. If all the contractive factors $\rho_{j}$ are equal, we say the equicontractive hypothesis holds. This is the case for the usual Cantor measure, with $\rho_{1}=\rho_{2}=\frac{1}{3}$. It would appear that the equicontractive case would be the simplest, and indeed we will treat this case first. However, it turns out that the "generic" case is simpler. We will show that $\lim _{R \rightarrow \infty} H(R)$ exists and is nonzero in the case that the contractive factors are exponentially incommensurable (not satisfying $\rho_{j}^{\nu_{j}}=\rho$ for fixed $\rho$ and integers $\nu_{j}$ ). 
Another goal of this paper is to generalize the notion of self-similarity to distributions. The existence and uniqueness for self-similar probability measures satisfying a given identity (1.4) are proved directly in $[\mathrm{H}]$ using the contractive mapping principle. We can extend the definition (1.6) to distributions $f$ by

$$
\left\langle f \circ S_{j}^{-1}, \varphi\right\rangle=\left\langle f, \varphi \circ S_{j}\right\rangle
$$

for any test function $\varphi$. Then the analogue of $(1.4)$ is

$$
f=\sum_{j=1}^{m} a_{j} f \circ S_{j}^{-1},
$$

but we relax the requirement that the weights $a_{j}$ be positive and allow them to be complex numbers. We do not assume (1.5) a priori, although it turns out that it is the first $\left(\mathrm{A}_{0}\right)$ of a set of algebraic conditions denoted $\left(\mathrm{A}_{k}\right)$, $k=0,1,2, \ldots$, that are involved in giving necessary and sufficient conditions for the existence of distributions of compact support satisfying $\left(1.4^{\prime}\right)$. The construction of solutions of $\left(1.4^{\prime}\right)$ which we give is rather indirect; in fact we first construct the Fourier transform $F$ of $f$. Since $F$ must be an entire function of exponential type, we can use power series to construct $F$. Only in special cases is $F$ given by an infinite product analogous to the expression for $\hat{\mu}$ in Part I. Uniqueness of solutions is not automatic, but the space of solutions (with compact support) is always finite dimensional.

The construction of self-similar distributions of compact support by means of the Fourier transform is given in $\S 2$. In $\S 3$ we study the asymptotic behavior of averages of these Fourier transforms in the equicontractive case, proving the type of asymptotic periodicity revealed in Figure 1. In $\S 4$ we extend the asymptotic periodicity to what may be called a fractal Plancherel formula for the Fourier transforms of distributions in $L^{2}(d \mu)$ for certain self-similar measures $\mu$. This result is a refinement of the results of [S1], but for a smaller class of measures. In $\S 5$ we extend the results of $\S \S 3$ and 4 to the nonequicontractive case. The results are similar if $\rho_{j}^{\nu_{j}}=\rho$ for integers $\nu_{j}$ (exponential commensurability), but in the opposite case (exponential incommensurability) we obtain a simplification in that the periodic terms become constants. This kind of dichotomy is apparently quite common in some problems in probability theory (see Feller [F, p. 348] or Lalley [Ly]). In $\S 6$ we discuss briefly a number of possible generalizations of our results: to self-affine distributions, to infinite sums in (1.4), to noncompactly supported distributions, and to $L^{p}$ norms in (1.1).

This paper is a natural continuation of our work in [S1-S3] and of Hutchinson's work $[\mathrm{H}]$, but it is written so that it can mainly be read independently. Somewhat different but related ideas in the context of Riemannian manifolds are given in [S4]. The numerical and graphical work to produce the figures was done by Maria Korolov.

Finally, we draw the reader's attention to two recent works which further develop some of the themes of this paper, [HL] and [Lu].

Note added in proof. Additional references which extend the results of this paper are [Lu2, LW and S6]. Related ideas are discussed in [DL, JRS, S5 and S7]. 


\section{SELF-SIMILAR Distributions}

A self-similar distribution on $\mathbf{R}^{n}$ is defined to be a distribution $f$ on $\mathbf{R}^{n}$ which satisfies an identity

$$
f=\sum_{j=1}^{m} a_{j} f \circ S_{j}^{-1}
$$

for a set of complex numbers $a_{j}$ and contractive similarities

$$
S_{j} x=\rho_{j} R_{j} x+b_{j},
$$

where $0<\rho_{j}<1, R_{j}$ is an orthogonal transformation, and $b_{j}$ is a vector in $\mathbf{R}^{n}$, and where $f \circ S_{j}^{-1}$ is defined by

$$
\left\langle f \circ S_{j}^{-1}, \varphi\right\rangle=\left\langle f, \varphi \circ S_{j}\right\rangle
$$

so as to be consistent with the definition for measures (not for functions).

We will also use the iterated version of $(2.1)$,

$$
f=\sum_{J \in \mathscr{Z}_{N}} a_{J} f \circ S_{J}^{-1}
$$

where $\mathscr{J}_{N}$ is the set of $N$-tuples $J=\left(j_{1}, \ldots, j_{N}\right)$ with $1 \leq j_{k} \leq m, a_{J}=$ $\prod_{k=1}^{N} a_{j_{k}}$, and $S_{J}=S_{j_{1}} \circ S_{j_{2}} \circ \cdots \circ S_{j_{N}}$, or more explicitly $S_{J} x=\rho_{J} R_{J} x+b_{J}$ with

$$
\rho_{J}=\prod_{k=1}^{N} \rho_{j_{k}}, \quad R_{J}=R_{j_{1}} \circ \cdots \circ R_{j_{N}},
$$

and

$$
\begin{aligned}
b_{J}= & b_{j_{1}}+\rho_{j_{1}} R_{j_{1}} b_{j_{2}}+\rho_{j_{1}} \rho_{j_{2}} R_{j_{1}} R_{j_{2}} b_{j_{3}} \\
& +\cdots+\left(\rho_{j_{1}} \cdots \rho_{j_{N-1}}\right)\left(R_{j_{1}} \cdots R_{j_{N-1}}\right) b_{j_{N}} .
\end{aligned}
$$

We will primarily be concerned with self-similar distributions of compact support. We denote by $F$ the Fourier transform of $f$, which in this case is an entire analytic function of exponential type. We note that $(2.1)$ is then equivalent to the condition

$$
F(x)=\sum_{j=1}^{m} a_{j} e^{i b_{j} \cdot x} F\left(\rho_{j} R_{j}^{*} x\right)
$$

and by iterating $(2.5)$ we obtain

$$
F(x)=\sum_{J \in \mathscr{J}_{N}} a_{J} e^{i b_{J} \cdot x} F\left(\rho_{J} R_{J}^{*} x\right) .
$$

In order to construct self-similar distributions of compact support satisfying (2.1) we will solve (2.5). If we can construct an entire analytic function $F$ which satisfies (2.5) on the power series level at the origin (i.e., both sides of (2.5) have the identical Taylor expansion at the origin) then $F$ satisfies (2.5) on all of $\mathbf{C}^{n}$. The following lemma then shows that $F=\hat{f}$ for some distribution of compact support, and then (2.5) implies $f$ satisfies (2.1). 
Lemma 2.1. If an entire analytic funtion $F$ satisfies (2.5) then $F$ is of exponential type.

Proof. Since both sides of (2.6) are analytic functions the identity holds on $\mathbf{C}^{n}$,

$$
F(x+i y)=\sum_{J \in \mathscr{J}_{N}} a_{J} e^{i b_{J} \cdot x} e^{-b_{J} \cdot y} F\left(\rho_{J} R_{J}^{*}(x+i y)\right),
$$

hence

$$
|F(x+i y)| \leq A^{N} e^{B|y|} \sup _{J \in \mathscr{J}_{N}}\left|F\left(\rho_{J} R_{J}^{*}(x+i y)\right)\right|,
$$

where $A=\sum_{j=1}^{m}\left|a_{j}\right|$ and $B=\sup _{J}\left|b_{J}\right|$, which is finite (see [H]). Now since $\rho=\sup _{j} \rho_{j}<1$ we have $\left|\rho_{J} R_{J}^{*} z\right| \leq \rho^{N}|z|$, and so if we choose

$$
N \geq \log |z| / \log \rho^{-1}
$$

we have $\left|\rho_{J} R_{J}^{*} z\right| \leq 1$, hence $|F(z)| \leq M A^{N} e^{B|y|}$, where $M$ is an upper bound for $|F(z)|$ on the unit ball of $\mathbf{C}^{n}$. But since $N$ is a multiple of $\log |z|$ the factor $A^{N}$ is of polynomial growth in $|z|$. Q.E.D.

This approach to constructing $f$ does not give us control of the support of $f$, but this follows automatically from (2.1). In fact let $K$ denote the compact selfsimilar set associated to the set of similarities $S_{1}, \ldots, S_{m}$ constructed in [H]. Then any distribution of compact support satisfying (2.1) must be supported in $K$. To see this let $K_{1}$ be any compact set that supports $f$. Then given any $\varepsilon>0$ there exists $N$ such that all the sets $S_{J} K_{1}$ for $J \in \mathscr{J}_{N}$ are within an $\varepsilon$-neighborhood of $K$. Thus if $\varphi$ is a test function supported outside an $\varepsilon$-neighborhood of $K$,

$$
\langle f, \varphi\rangle=\sum_{J \in \mathscr{F}_{N}} a_{J}\left\langle f, \varphi \circ S_{J}\right\rangle=0
$$

since $\varphi \circ S_{J}(x)=0$ for $x$ in $K_{1}$. Thus supp $f \subseteq K$. Of course this is not to say that $f$ cannot be supported on a smaller set.

We turn now to the problem of constructing solutions of (2.5). Certain algebraic conditions on the weights $a_{j}$ (for given $\rho_{j}$ and $R_{j}$ ) will determine when this is possible. We call these conditions $\left(\mathrm{A}_{k}\right)$, for $k=0,1,2, \ldots$. The condition $\left(A_{0}\right)$ is simply

$$
\sum_{j=1}^{m} a_{j}=1
$$

and $\left(A_{1}\right)$ is

$$
I-\sum_{j=1}^{m} a_{j} \rho_{j} R_{j} \quad \text { is not invertible. }
$$

To state the higher order conditions we define $R_{j}^{(k)}=R_{j} \otimes \cdots \otimes R_{j} \quad(k$ factors) to be the extension of $R_{j}$ to an operator on the $k$-fold symmetric tensors on $\mathbf{R}^{n}$. If we represent this space of tensors by the homogeneous polynomials $p_{k}(x)$ of degree $k$ on $\mathbf{R}^{n}$, then the action of $R_{j}^{(k)}$ is just $p_{k}(x) \rightarrow p_{k}\left(R_{j}^{*} x\right)$. Then $\left(\mathbf{A}_{k}\right)$ for $k \geq 2$ is

$$
I-\sum_{j=1}^{m} a_{j} \rho_{j}^{k} R_{j}^{(k)} \text { is not invertible. }
$$


It is clear that this is a generalization of $\left(A_{0}\right)$ and $\left(A_{1}\right)$, and it is clearly an algebraic condition since it is equivalent to the vanishing of a determinant of a matrix whose entries are linear in $a_{j}$.

Theorem 2.2. The space of analytic solutions to (2.5) is at most finite dimensional, and nontrivial solutions exist if and only if one of the conditions $\left(\mathrm{A}_{k}\right)$ holds. If $\left(\mathrm{A}_{0}\right)$ holds but none of the other $\left(\mathrm{A}_{k}\right)$ conditions hold, then the space of solutions is exactly one-dimensional and there is a unique solution satisfying $F(0)=1$. If $k^{\prime}$ denotes the largest value of $k$ for which $\left(\mathrm{A}_{k}\right)$ holds, then the dimension of the space of solutions is at least the nullity of $I-\sum_{j=1}^{m} a_{j} \rho_{j}^{k^{\prime}} R_{j}^{\left(k^{\prime}\right)}$, and at most the dimension of the space of polynomials of degree $\leq k^{\prime}$ in $\mathbf{R}^{n}$, with the exact value depending on the values of $b_{j}$.

Proof. Observe that at most a finite number of conditions $\left(\mathrm{A}_{k}\right)$ can hold (for fixed $\left.a_{j}, \rho_{j}, R_{j}\right)$, because for $k$ sufficiently large the operator $\sum_{j=1}^{m} a_{j} \rho_{j}^{k} R_{j}^{(k)}$ has norm less than one.

Now if $F$ is analytic then it has a convergent power series expansion $\sum_{k=0}^{\infty} P_{k}(x)$ about the origin, where

$$
P_{k}(x)=\sum_{|\alpha|=k} c_{\alpha} x^{\alpha}
$$

is a homogeneous polynomial of degree $k$. Substituting this expansion in (2.5) we find

$$
P_{k}(x)-\sum_{j=1}^{m} a_{j} \rho_{j}^{k} P_{j}\left(R_{j}^{*} x\right)=\sum_{j=1}^{m} \sum_{l=1}^{k} a_{j} \frac{\left(i b_{j} \cdot x\right)^{l}}{l !} \rho_{j}^{k-l} P_{k-l}\left(R_{j}^{*} x\right) .
$$

If condition $\left(\mathrm{A}_{k}\right)$ fails then we can obtain $P_{k}(x)$ from the left side of (2.7). Thus if $P_{k}=0$ for all $k \leq k^{\prime}$, then (2.7) implies inductively that all $P_{k} \equiv 0$, hence $F=0$. This shows that the space of solutions is always finite dimensional, with an upper bound on the dimension given by the dimension of the space of polynomials of degree $\leq k^{\prime}$. In particular, if none of the conditions $\left(\mathrm{A}_{k}\right)$ holds then there are no nontrivial solutions, while if $k^{\prime}=0$ then the space of solutions is at most one-dimensional.

Conversely, assume that $\left(\mathrm{A}_{k}\right)$ holds for at least one value of $k$, with $k^{\prime}$ the largest of these. Let $p(x)$ be any polynomial homogeneous of degree $k^{\prime}$ such that

$$
p(x)-\sum_{j=1}^{m} a_{j} \rho_{j}^{k} p\left(R_{j}^{*} x\right)=0 .
$$

If we set $P_{k}=0$ for $k<k^{\prime}$ and $P_{k^{\prime}}=p$ then (2.7) is valid for $k \leq k^{\prime}$, and then we can use (2.7) to solve inductively for $P_{k}$ for all $k$. This gives us a formal power series solution of (2.5). However it is a routine matter to show that the form of $(2.7)$ forces the coefficients to decay so that the power series has infinite radius of convergence. Thus we obtain entire analytic solutions to (2.5) with prescribed Taylor expansion up to order $k^{\prime}$. If $k^{\prime}=0$ we can take $F(0)=1$. If $k^{\prime}>0$ and there are other values of $k$ for which $\left(\mathrm{A}_{k}\right)$ holds, we can try to prescribe nonzero values for those $P_{k}$, but whether or not this leads to a formal solution to (2.7) depends on the coefficients $b_{j}$ satisfying certain complicated polynomial identities. Q.E.D. 
As an example of what can happen when $\left(A_{k}\right)$ holds for more than one value of $k$, consider the equation

$$
F(x)=4 F\left(\frac{1}{2} x\right)-3 e^{i b x} F\left(\frac{1}{3} x\right)
$$

in $\mathbf{R}^{1}$. When $b=0$ there is a two-dimensional space of solutions, $F(x)=$ $c x+d$, but when $b \neq 0$ we must have $F(0)=0$ and there is only a onedimensional space of solutions.

When $n=1$ we can essentially reduce the construction in the theorem to the case when $\left(\mathrm{A}_{0}\right)$ holds but no $\left(\mathrm{A}_{k}\right)$ holds for $k \geq 1$. Indeed, suppose $k^{\prime}$ in the theorem is greater than zero. Then by setting $F(x)=x^{k^{\prime}} G(x)$ we find that $G$ must satisfy a version of $(2.5)$ in which $\left(\mathrm{A}_{0}\right)$ holds but no $\left(\mathrm{A}_{k}\right)$ holds for $k \geq 1$. Of course this may not yield all solutions to the original problem, and such a simplification does not work in higher dimensions.

From now on we assume that $\left(\mathrm{A}_{0}\right)$ holds. There are three special cases in which we can immediately conclude that no $\left(\mathrm{A}_{k}\right)$ can hold for $k \geq 1$. The first case is when the weights are positive, $0 \leq a_{j} \leq 1$. For then

$$
\left\|\sum_{j=1}^{m} a_{j} \rho_{j}^{k} R_{j}^{(k)}\right\| \leq \sum_{j=1}^{m} a_{j} \rho_{j}^{k}<1
$$

since $\left\|R_{j}^{(k)}\right\|=1$. This is the situation we considered in Part I which led to the construction of self-similar measures. Thus the uniqueness of self-similar measures is valid even in the class of distributions with compact support. The second case is when all the similarities $S_{j}$ have the same linear part, i.e., all $R_{j}=R$ and all $\rho_{j}=\rho$. For then

$$
\left\|\sum_{j=1}^{m} a_{j} \rho_{j}^{k} R_{j}^{(k)}\right\|=\rho^{k}\left\|R^{(k)}\right\|=\rho^{k}<1 .
$$

The third case is when

$$
\sum_{j=1}^{m}\left|a_{j}\right| \rho_{j}<1
$$

which of course implies

$$
\sum_{j=1}^{m}\left|a_{j}\right| \rho_{j}^{k}<1 \quad \text { for all } k \geq 1
$$

and then

$$
\left\|\sum_{j=1}^{m} a_{j} \rho_{j}^{k} R_{j}^{(k)}\right\| \leq \sum_{j=1}^{m}\left|a_{j}\right| \rho_{j}^{k}<1 .
$$

Of course this case contains the first case.

In all these cases there is a more satisfactory description of the unique solution of (2.5) with $F(0)=1$. Let us write

$$
F_{N}(x)=\sum_{J \in \mathscr{f}_{N}} a_{J} e^{i b_{J} \cdot x}
$$


In the first case we showed in Part I that $F_{N}(x) \rightarrow F(x)$ uniformly on compact sets (under certain restricted conditions, which are not necessary for this conclusion). We will show this is true in all these cases. Note that in the second case we can write

$$
F_{N}(x)=\prod_{k=0}^{N-1} F_{1}\left(\rho^{k}\left(R^{*}\right)^{k} x\right) .
$$

Theorem 2.3. Let $\left(\mathrm{A}_{0}\right)$ hold and assume either (i) (2.8) holds, or (ii) all $S_{j}$ have the same linear part. Then

$$
F(x)=\lim _{N \rightarrow \infty} F_{N}(x)
$$

uniformly on compact sets.

Proof. In case (i) we use (2.6) and (2.9) to obtain

$$
F(x)-F_{N}(x)=\sum_{J \in \mathscr{F}_{N}} a_{J} e^{i b_{J} \cdot x}\left(F\left(\rho_{J} R_{J}^{*} x\right)-1\right)
$$

and hence

$$
\left|F(x)-F_{N}(x)\right| \leq \sum_{J \in \mathscr{J}_{N}}\left|a_{J}\right|\left|F\left(\rho_{J} R_{J}^{*} x\right)-1\right| .
$$

Since $F(0)=1$ we have

$$
|F(x)-1| \leq c|x| \text { if }|x| \leq 1 .
$$

Then if $|x| \leq \rho^{-N}$ (recall $\rho$ is the maximum of $\rho_{j}$ ) we have

$$
\left|F(x)-F_{N}(x)\right| \leq c \sum_{J \in \mathscr{f}_{N}}\left|a_{J}\right| \rho_{J}|x| \leq c\left(\sum_{j=1}^{m}\left|a_{j}\right| \rho_{j}\right)^{N}|x|,
$$

which proves $(2.11)$.

In case (ii) we use (2.10). First we show that the infinite product

$$
F_{\infty}(x)=\lim _{N \rightarrow \infty} F_{N}(x)=\prod_{k=0}^{\infty} F_{1}\left(\rho^{k}\left(R^{*}\right)^{k} x\right)
$$

exists uniformly on compact sets. But this follows from the convergence of

$$
\sum_{k=0}^{\infty}\left|F_{1}\left(\rho^{k}\left(R^{*}\right)^{k} x\right)-1\right|
$$

which follows from the estimate $\left|F_{1}(x)-1\right| \leq c|x|$. Once we have the existence of $F_{\infty}$ it is easy to show that it satisfies (2.5), and since $F_{\infty}(0)=1$ we have $F=F_{\infty}$ by the uniqueness part of Theorem 2.2. Q.E.D.

\section{ASYMPTOTIC PERIODICITY}

Let $f$ be a self-similar distribution of compact support with Fourier transform $F$. Throughout this section we make the equicontractive hypothesis that 
all $\rho_{j}$ are equal, and we denote this common value by $\rho$. We also assume $\left(\mathrm{A}_{0}\right)$ holds and we define the real number $\beta$ by

$$
\rho^{\beta}=\sum_{j=1}^{m}\left|a_{j}\right|^{2} .
$$

This is consistent with our notation in Part I, except that now we do not assert that $\beta$ is positive; however the argument in Part I that $\beta \leq n$ under the open set condition is still valid. We are interested in the asymptotic behavior of the averages

$$
H(R)=\frac{1}{R^{n-\beta}} \int_{|x| \leq R}|F(x)|^{2} d x
$$

as $R \rightarrow \infty$, and we will show that $H(R)$ tends in the limit to a function that is periodic in $\log R$. In order to do this we first study the averages

$$
h(t)=t^{(n-\beta) / 2} \int|F(x)|^{2} e^{-t|x|^{2}} d x
$$

as $t \rightarrow 0$, and then use a Tauberian theorem to obtain information about $H(R)$.

Now for $h(t)$ the periodicity involves comparing it with $h\left(\rho^{-2} t\right)$. The following computations give the basic idea. On the one hand, if we use the definition of $\beta$ and a change of variable we find

$$
\begin{aligned}
h\left(\rho^{-2} t\right) & =\rho^{\beta-n} t^{(n-\beta) / 2} \int e^{-t \rho^{-2}|x|^{2}}|F(x)|^{2} d x \\
& =\sum_{j=1}^{m}\left|a_{j}\right|^{2} \rho^{-n} t^{(n-\beta) / 2} \int e^{-t \rho^{-2}|x|^{2}}|F(x)|^{2} d x \\
& =\sum_{j=1}^{m}\left|a_{j}\right|^{2} t^{(n-\beta) / 2} \int e^{-t|x|^{2}}\left|F\left(\rho R_{j}^{*} x\right)\right|^{2} d x .
\end{aligned}
$$

On the other hand, if we use (2.5) and expand we obtain

$$
\begin{aligned}
h(t)= & t^{(n-\beta) / 2} \int e^{-t|x|^{2}}\left|\sum_{j=1}^{m} a_{j} e^{i b_{j} \cdot x} F\left(\rho R_{j}^{*} x\right)\right|^{2} d x \\
= & \left.\sum_{j=1}^{n_{1}}\left|a_{j}\right|^{2} t^{(n-\beta) / 2} \int e^{-t|x|^{2}} \mid F\left(\rho R_{j}^{*} x\right)\right)\left.\right|^{2} d x \\
& +\sum_{j \neq k} a_{j} \bar{a}_{k} t^{(n-\beta) / 2} \int e^{-t|x|^{2}} e^{i\left(b_{j}-b_{k}\right) \cdot x} F\left(\rho R_{j}^{*} x\right) \overline{F\left(\rho R_{k}^{*} x\right)} d x .
\end{aligned}
$$

Comparing (3.4) and (3.5) we can write

$$
h(t)-h\left(\rho^{-2} t\right)=E(t),
$$

where

$$
E(t)=\sum_{j \neq k} a_{j} \bar{a}_{k} E_{j k}(t)
$$


and

$$
E_{j k}(t)=t^{(n-\beta) / 2} \int e^{-t|x|^{2}} e^{i\left(b_{j}-b_{k}\right) \cdot x} F\left(\rho R_{j}^{*} x\right) \overline{F\left(\rho R_{k}^{*} x\right)} d x .
$$

Now the idea is that $E(t)$ is a small error term, and to show this it suffices to show the same for each $E_{j k}(t), j \neq k$. But since $e^{i b_{j} \cdot x} F\left(\rho R_{j}^{*} x\right)$ is the Fourier transform of $f \circ S_{j}^{-1}$, we have

$$
E_{j k}(t)=\pi^{n / 2} t^{-\beta / 2} \iint e^{-|x-y|^{2} / 4 t} f \circ S_{j}^{-1}(x) \overline{f \circ S_{k}^{-1}(y)} d x d y
$$

(the integrals here are used in a symbolic sense, since $f$ is only a distribution). We can also write this as

$$
E_{j k}(t)=\left\langle f \circ S_{j}^{-1} * \overline{f \circ S_{k}^{-1}}, \psi_{t}\right\rangle,
$$

where

$$
\psi_{t}(x)=\pi^{n / 2} t^{-\beta / 2} e^{-|x|^{2} / 4 t} .
$$

At this point we need to use the essential disjointness of the distributions $f \circ S_{j}^{-1}$ and $f \circ S_{k}^{-1}$. Unfortunately, the open set condition of [H] just fails to give us what we need, so we use a stronger hypothesis on the set of similarities.

Definition 3.1. We say the open set condition holds if there exists a bounded open set $U$ such that $S_{j} U \subseteq U$ for each $j$, and $S_{j} U$ and $S_{k} U$ are disjoint for each $j \neq k$. We say the strong open set condition holds if in addition the closures of $S_{j} U$ and $S_{k} U$ are disjoint for $j \neq k$.

Under the strong open set condition the distribution $f \circ S_{j}^{-1}$ has support in $\overline{S_{j} U}$ which is disjoint from the support of $f \circ S_{k}^{-1}$, so the distribution $f \circ S_{j}^{-1} * \overline{f \circ S_{k}^{-1}}$ vanishes in a neighborhood of the origin.

Lemma 3.2. Assume the strong open set condition. Then

$$
\lim _{q \rightarrow \infty} h\left(\rho^{2 q} t\right)=\tilde{h}(t)
$$

exists uniformly on compact subsets of $(0, \infty)$.

Proof. We need to estimate

$$
h\left(\rho^{2 q_{1}} t\right)-h\left(\rho^{2 q_{2}} t\right)=\sum_{q=q_{1}+1}^{q_{2}} E\left(\rho^{2 q} t\right)
$$

uniformly in $t, q_{1}$, and $q_{2}$. Clearly it suffices to estimate

$$
\sum_{q=q_{1}+1}^{q_{2}} E_{j k}\left(\rho^{2 q} t\right)=\left\langle f \circ S_{j}^{-1} * \overline{f \circ S_{k}^{-1}}, \sum_{q=q_{1}+1}^{q_{2}} \psi_{\rho^{2 q t}}\right\rangle \text {. }
$$

Since $f \circ S_{j}^{-1} * \overline{f \circ S_{k}^{-1}}$ is a distribution of compact support vanishing in a neighborhood of the origin, it satisfies an estimate of the form

$$
\left|\left\langle f \circ S_{j}^{-1} * \overline{f \circ S_{k}^{-1}}, \varphi\right\rangle\right| \leq c \sum_{|\alpha| \leq N} \sup _{|x| \geq \varepsilon}\left|\left(\frac{\partial}{\partial x}\right)^{\alpha} \varphi(x)\right| .
$$


Thus we need to estimate

$$
\sup _{|x| \geq \varepsilon}\left|\left(\frac{\partial}{\partial x}\right)^{\alpha} \sum_{q=q_{1}+1}^{q_{2}} \psi_{\rho^{2 q} t}(x)\right| .
$$

But from (3.11) it follows easily that this can be made as small as we please (uniformly in $0<a \leq t \leq b$ ) provided we take $q_{1}$ and $q_{2}$ large enough. This establishes (3.12). Q.E.D.

Theorem 3.3. Let $f$ be any self-similar distribution of compact support with weights satisfying $\left(\mathrm{A}_{0}\right)$ and similarities satisfying the equicontractive condition and the strong open set condition. Let $f$ denote the Fourier transform of $f$ and let $h(t)$ be given by (3.3). Then there exists a bounded continuous function $\tilde{h}(t)$ on $(0, \infty)$ satisfying

$$
\tilde{h}\left(\rho^{2} t\right)=\tilde{h}(t)
$$

(in fact $\tilde{h}$ is given by (3.12)) such that

$$
\lim _{t \rightarrow 0}(h(t)-\tilde{h}(t))=0 .
$$

Proof. Define $\tilde{h}(t)$ by (3.12). Then (3.13) is obvious. Since $\tilde{h}$ is the uniform limit of continuous functions it is continuous, and together with the periodicity (3.13) this shows $\tilde{h}$ is bounded. For any $t<1$ we can write $t=\rho^{2 q} t_{0}$ with $\rho^{2} \leq t_{0} \leq 1$. Then $h(t)-\tilde{h}(t)=h\left(\rho^{2 q} t_{0}\right)-\tilde{h}\left(t_{0}\right)$ and $q \rightarrow \infty$ as $t \rightarrow 0$, so (3.12) implies (3.14). Q.E.D.

Remark. The proof actually shows that the rate of convergence in (3.14) is extremely rapid, namely

$$
h(t)=\tilde{h}(t)+O\left(e^{-\varepsilon / t}\right) \quad \text { as } t \rightarrow 0 .
$$

This follows easily from the estimate

$$
\sup _{|x| \geq \varepsilon}\left|\left(\frac{\partial}{\partial x}\right)^{\alpha} \psi_{t}(x)\right|=O\left(e^{-\varepsilon / t}\right)
$$

(here we are using $\varepsilon$ to denote a positive quantity that may vary from place to place).

Corollary 3.4. Let the hypotheses of Theorem 3.3 hold, and let $\beta<n$. Then there exists a bounded function $\widetilde{H}(R)$ satisfying

$$
\widetilde{H}(\rho R)=\widetilde{H}(R)
$$

such that $H(R)=\widetilde{H}(R)+E(R)$ with

$$
\lim _{R \rightarrow \infty} \int_{R \rho}^{R}|E(r)| \frac{d r}{r}=0,
$$

where $H(R)$ is given by (3.2).

Proof. We will apply Wiener's second Tauberian theorem [W, p. 74, Theorem 5] (or [T, p. 338, Theorem 7.6] for a more concise exposition). Let $f(s)=$ $e^{\beta s} \int_{S^{n-1}}\left|F\left(e^{s} u\right)\right|^{2} d u$. Then a change of variable shows $h\left(e^{-2 x}\right)=K_{1} * f(x)$ and $H\left(e^{x}\right)=K_{2} * f(x)$, where $K_{1}(x)=e^{(\beta-n) x} e^{-e^{-2 x}}$ and $K_{2}(x)=e^{(\beta-n) x} \chi_{x \geq 0}$ 
(one-dimensional convolution). Because $K_{2}$ is discontinuous we introduce a variant $K_{3}$, which is obtained by modifying $K_{2}$ near the origin to make it continuous.

$$
K_{3}(x)= \begin{cases}e^{(\beta-n) x} & \text { if } x \geq 0, \\ \delta^{-1} x+1 & \text { if }-\delta<x<0, \\ 0 & \text { if } x \leq-\delta,\end{cases}
$$

where $\delta$ is fixed (but later we will let $\delta \rightarrow 0$ ). In order to apply the Tauberian theorem we need to show that $K_{1}$ and $K_{3}$ are continuous and satisfy

$$
\sum_{j=-\infty}^{\infty} \sup \{|K(x)|: j \leq x \leq j+1\}<\infty
$$

and that $\hat{K}_{1}$ never vanishes. But (3.17) is routine, and a change of variable shows that

$$
\hat{K}_{1}(y)=\frac{1}{2} \int_{0}^{\infty} s^{(n-\beta-i y) / 2-1} e^{-s} d s=\frac{1}{2} \Gamma\left(\frac{n-\beta-i y}{2}\right),
$$

where $\Gamma$ never vanishes. The Tauberian theorem then implies that $K_{3}$ may be approximated in the norm given by (3.17) by linear combinations of translates of $K_{1}$.

Next we need to show that $f$ belongs to the dual space of the continuous functions satisfying (3.17), namely

$$
\sup _{k} \int_{k}^{k+1}|f(s)| d s<\infty .
$$

Now $h(t)$ is uniformly bounded: for $t \leq 1$ this follows from Theorem 3.3, while for $t \geq 1$ this follows from the definition and the fact that $F(x)$ has polynomial growth. Then

$$
\int_{k}^{k+1}|f(s)| d s=\int_{e^{k} \leq|x| \leq e^{k+1}}|F(x)|^{2}|x|^{\beta-n} d x<\operatorname{ch}\left(e^{-2 k}\right),
$$

which establishes (3.18).

Thus $K_{3} * f(x)$ is a uniform limit of linear combinations of translates of $K_{1} * f$. Now Theorem 3.3 says that $K_{1} * f$ is asymptotically periodic (the sum of a bounded continuous periodic function of period $\rho$ plus a function vanishing as $x \rightarrow \infty)$. Since the class of asymptotically periodic functions is closed under uniform limits, it follows that $K_{3} * f$ is also asymptotically periodic. In order to compare $K_{2} * f$ and $K_{3} * f$ we introduce $K_{4}$,

$$
K_{4}(x)= \begin{cases}1-\delta^{-1}|x| & \text { if }|x|<\delta, \\ 0 & \text { otherwise }\end{cases}
$$

and note that

$$
\left|K_{2} * f(x)-K_{3} * f(x)\right| \leq K_{4} * f(x) .
$$

We can apply the Tauberian theorem to the kernels $K_{1}$ and $K_{4}$ to show that $K_{4} * f(x)$ is the uniform limit of functions of the form $\sum c_{j} K_{1} * f\left(x+y_{j}\right)$. Now if $K_{1} * f(x)=P(x)+E(x)$, where $P(x+\rho)=P(x)$ and $E(x) \rightarrow 0$ as $x \rightarrow \infty$, 
this means $K_{4} * f(x)$ is the uniform limit of $\sum c_{j} P\left(x+y_{j}\right)+\sum c_{j} E\left(x+y_{j}\right)$. We integrate over a period in order to simplify the first term,

$$
\int_{a}^{a+\beta} K_{4} * f(x) d x \approx\left(\sum c_{j}\right) \int_{0}^{\rho} P(x) d x+\sum c_{j} \int_{a}^{a+\rho} E\left(x+y_{j}\right) d x .
$$

The point of doing this is that we can control $\sum c_{j}$ even though we cannot control the individual constants $c_{j}$. In fact $K_{4}$ is the limit in the (3.17) norm, hence the $L^{1}$ norm, of $\sum c_{j} K_{1}\left(x+y_{j}\right)$, hence

$$
\left|\sum c_{j}\right| \leq 2\left\|K_{1}\right\|_{1}^{-1}\left\|K_{4}\right\|_{1}
$$

and we can make $\left\|K_{4}\right\|_{1} \rightarrow 0$ by letting $\delta \rightarrow 0$.

Since $\sum c_{j}$ is multiplied by $\int_{0}^{\rho} P(x) d x$, which is a fixed constant in (3.20), we can arrange to make $\int_{a}^{a+\rho} K_{4} * f(x) d x$ equal to the sum of an arbitrarily small function plus a function vanishing as $x \rightarrow \infty$ by making $\delta$ sufficiently small.

Now as $\delta \rightarrow 0, K_{3} * f$ decreases monotonically to $K_{2} * f$. Thus if $K_{3} * f=$ $P_{\delta}+E_{\delta}$ with $P_{\delta}$ periodic then $P_{\delta}$ decreases monotonically to a limit $P$ which is bounded and periodic. A routine argument using (3.19) and the properties of $K_{4} * f$ shows that $K_{2} * f=P+E$, where

$$
\lim _{a \rightarrow \infty} \int_{a}^{a+\rho}|E(x)| d x=0 .
$$

Finally, a change of variable transforms (3.21) into (3.16). Q.E.D.

Note added in proof. An improvement of this result is given in [LW].

While the passage from $\tilde{h}$ to $\widetilde{H}$ is complicated, in the reverse direction it is easy to see that

$$
h(t)=2 t^{(n-\beta) / 2+1} \int_{0}^{\infty} R^{n-\beta+1} e^{-t R^{2}} H(R) d R,
$$

hence

$$
\tilde{h}(t)=2 t^{(n-\beta) / 2+1} \int_{0}^{\infty} R^{n-\beta+1} e^{-t R^{2}} \widetilde{H}(R) d R .
$$

In the special case that $f$ is a measure (all weights $a_{j}$ are positive) and $\beta<$ $(n+1) / 2$ we can avoid using the Tauberian theorem by giving a direct proof of the corollary, imitating the proof of the theorem. This gives uniform convergence, and in fact a rate of convergence, but not as fast as $\left(3.14^{\prime}\right)$.

Corollary 3.5. Let $f$ be a measure satisfying the hypotheses of Theorem 3.3, and assume $\beta<(n+1) / 2$. Then

$$
H(R)=\widetilde{H}(R)+O\left(R^{\beta-((n+1) / 2)}\right) \quad \text { as } R \rightarrow \infty .
$$

Proof. Imitating the proof of Lemma 3.2 we find $H(R)-H(\rho R)=\widetilde{E}(R)$, where $\widetilde{E}(R)=\sum_{j \neq k} a_{j k} \widetilde{E}_{j k}(R)$ and

$$
\begin{aligned}
\widetilde{E}_{j k}(R) & =R^{\beta-n} \int_{|x| \leq R} e^{i\left(b_{j}-b_{k}\right) \cdot x} F\left(\rho R_{j}^{*} x\right) \overline{F\left(\rho R_{k}^{*} x\right)} d x \\
& =c R^{\beta-n / 2} \iint J_{n / 2}(R|x-y|)|x-y|^{-n / 2} d \mu_{j}(x) d \bar{\mu}_{k}(y),
\end{aligned}
$$


where $\mu_{j}=f \circ S_{j}^{-1}, \mu_{k}=f \circ S_{k}^{-1}$, and $J_{n / 2}$ is the Bessel function. Since the supports of $\mu_{j}$ and $\mu_{k}$ are separated, we have $|x-y| \geq \varepsilon$ in the integral and we use standard estimates on the Bessel function to obtain

$$
\left|J_{n / 2}(R|x-y|)\right| x-\left.y\right|^{-n / 2} \mid \leq c R^{-1 / 2},
$$

hence $\left|\widetilde{E}_{j k}(R)\right| \leq c R^{\beta-((n+1) / 2)}$. Since $\beta-((n+1) / 2)<0$ by hypothesis, the rest of the proof proceeds as before. Q.E.D.

Theorem 3.6. Under the hypothesis of Theorem 3.3, either $\widetilde{H}$ is identically zero or $\widetilde{H}$ is never zero, and

$$
\min \widetilde{H} \geq \rho^{n-\beta} \max \tilde{H} .
$$

Proof. Let $R$ be a point where $\widetilde{H}$ assumes its minimum, and $a R$ a point where $\widetilde{H}$ assumes its maximum, with $\rho \leq a \leq 1$. Then $H(R) \geq a^{n-\beta} H(a R)$ follows from

$$
\int_{|x| \leq R}|F(x)|^{2} d x \geq \int_{|x| \leq a R}|F(x)|^{2} d x
$$

and so

$$
\widetilde{H}(R) \geq a^{n-\beta} \widetilde{H}(a R)+\widetilde{E}(R),
$$

where $\widetilde{E}(R)=(\widetilde{H}(R)-H(R))+a^{n-\beta}(H(a R)-\widetilde{H}(a R))$. But the same is true if we replace $R$ by $\rho^{-k} R$, so

$$
\widetilde{H}(R) \geq a^{n-\beta} \widetilde{H}(a R)+\widetilde{E}\left(\rho^{-k} R\right) .
$$

But $\widetilde{E}\left(\rho^{-k} R\right) \rightarrow 0$ as $k \rightarrow \infty$ so $\widetilde{H}(R) \geq a^{n-\beta} \widetilde{H}(a R)$, which gives (3.25) since $\rho \leq a$ and $n-\beta \geq 0$. Finally, if $\widetilde{H}$ ever vanishes then (3.25) implies $\widetilde{H}$ is identically zero. Q.E.D.

Of course the case when $\widetilde{H}$ never vanishes is the more interesting alternative, and it is desirable to have conditions which imply that it holds. In $\S 5$ we will give a general result to this effect, under the assumption that the weights $\left\{a_{j}\right\}$ are all positive. Without this assumption we require some severe restrictions on the similarities.

Corollary 3.7. Assume that the similarities $S_{j}$ satisfy the following conditions for some fixed integer $r \geq 3: \rho=1 / r$, each component of $r b_{j}$ is an integer between 0 and $r-1$, and all $R_{j}$ are equal to an orthogonal transformation that preserves the integer lattice. Assume also the strong open set condition (in this case $\left.U=\left\{x: 0<x_{j}<1, j=1, \ldots, n\right\}\right)$. Assume that the weights satisfy $\left(\mathrm{A}_{0}\right)$, and finally assume that there exists a fundamental domain $D$ (for the torus $\mathbf{R} / 2 \pi \mathbf{Z}^{n}$ ) containing a neighborhood of the origin on which $|F(x)|$ is bounded away from zero. Then $\widetilde{H}(R)$ never vanishes.

Proof. The argument given in the proof of Theorem 4.4 in Part I shows

$$
\liminf _{R \rightarrow \infty}\left(R^{n-\beta}\right)^{-1} \int_{|x| \leq R}|F(x)|^{2} d x
$$

is positive, and this easily implies $\widetilde{H}(R)$ never vanishes. Q.E.D.

In Part I we showed that the existence of $D$ always holds when $n=1$ or 2 in the case of measures, but that argument is not valid for distributions since it 
requires the positivity of the weights. However, if we assume the hypotheses of Theorem 2.2 then this condition is in some sense generically fulfilled. For then, since (2.11) holds, $|F(x)|$ will be bounded away from zero on any compact set provided $F_{1}(x)$ given by $(2.9)$ never vanishes. Now $F_{1}(x)$ is just a periodic trigonometric polynomial with fixed frequencies, and amplitudes given by the weights. The $\left(\mathrm{A}_{0}\right)$ condition implies $F_{1}(0)=1$ but does not force $F_{1}$ to vanish anywhere. It is then straightforward to show that there exists an open dense subset of the subspace of $\mathbf{C}^{m}$ of weights satisfying $\left(\mathrm{A}_{0}\right)$ for which $F_{1}(x)$ never vanishes.

The hypothesis that the similarities satisfy the strong open set condition seems excessive, and we conjecture that all the results of this section hold under the open set condition. As a step in this direction we give an argument that works for measures under an auxiliary condition which is satisfied in a large number of examples. Since the difference between the two conditions involves the possible intersection of the closures of $S U_{j}$ and $S U_{k}$, we will need to assume that this intersection has measure zero. In addition, we need the condition

$$
\int_{S U_{j}} \int_{S U_{k}}|x-y|^{-\beta} d \mu_{j}(x) d \mu_{k}(y)<\infty \text { for } j \neq k,
$$

where $\mu_{j}=\mu \circ S_{j}^{-1}, \mu_{k}=\mu \circ S_{k}^{-1}$, and we have written $\mu$ in place of $f$.

Lemma 3.8. Let $\mu$ be a self-similar measure satisfying the equicontractive and open set conditions, such that $\mu\left(\overline{S_{j} U} \cap \overline{S_{k} U}\right)=0$ for $j \neq k$ and (3.26) holds. Then the conclusions of Theorem 3.3 and Corollary 3.4 hold.

Proof. The only essential change is in the estimate of (3.9), which in our notation is now

$$
\pi^{n / 2} t^{-\beta / 2} \iint e^{-|x-y|^{2} / 4 t} d \mu_{j} d \mu_{k} .
$$

We want to use the dominated convergence theorem to show this goes to zero as $t \rightarrow 0$. The integrand tends to zero if $x \neq y$, and this is almost everywhere because of the hypothesis $\mu\left(\overline{S_{j} U} \cap \overline{S_{k} U}\right)=0$. The integrand is dominated by a multiple of $|x-y|^{-\beta}$, and (3.26) says exactly that the dominator is integrable. Q.E.D.

Suppose now that $n=1$, the similarities $S_{j} x=\rho x+b_{j}$, and the open set condition holds for $U$ an interval. Then $S_{j} U$ and $S_{k} U$ are disjoint open intervals so the intersection of the closures is at most a point. Since it is easy to see that $\mu$ is nonatomic, we have $\mu\left(\overline{S_{j} U} \cap \overline{S_{k} U}\right)=0$. We claim also that (3.26) holds.

To see this assume $\overline{S_{j} U} \cap \overline{S_{k} U}$ intersect in a point $A$ (otherwise (3.26) is trivial) with $S_{j} U$ on the left and $S_{k} U$ on the right. There is at most one similarity, call it $S_{1}$, which maps the left endpoint of $U$ to itself, and similarly $S_{2}$ which maps the right endpoint of $U$ to itself (if both $S_{1}$ and $S_{2}$ do not exist then (3.26) is again trivial). Let $p$ denote an index that varies in $(2,3, \ldots, m)$, and let $q$ vary in $(1,3, \ldots, m)$. Thus $S_{1} S_{k} U$ and $S_{2} S_{j} U$ meet at $A$, but $S_{p} S_{k} U$ lies at a distance of at least $\rho^{2}$ from $S_{j} U$, and $S_{q} S_{j} U$ lies at a distance of at least $\rho^{2}$ from $S_{k} U$ (we assume $U$ has length one). 
Now we subdivide $S_{k} U$ into the disjoint intervals $S_{p} S_{1}^{l} S_{k} U$ for $l=0,1$, $2, \ldots, p=2, \ldots, m$ and denote by $\mu_{p, l, k}$ the restriction of $\mu_{k}$ to $S_{p} S_{1}^{l} S_{k} U$. It is clear that $\mu_{k}=\sum_{p, l} \mu_{p, l, k}$. Similarly, $\mu_{j}=\sum_{q, l^{\prime}} \mu_{q, l^{\prime}, j}$, where $\mu_{q, l^{\prime}, j}$ is the restriction of $\mu_{j}$ to $S_{q} S_{2}^{l^{\prime}} S_{j} U$. Then

$$
\iint|x-y|^{-\beta} d \mu_{k}(x) d \mu_{j}(y)=\sum_{p, l} \sum_{q, l^{\prime}} \iint|x-y|^{-\beta} d \mu_{p, l, k}(x) d \mu_{q, l^{\prime}, j}(y) .
$$

The total measure of $\mu_{p, l, k}$ is $a_{p} a_{1}^{l}$, and the total measure of $\mu_{q, l^{\prime}, j}$ is $a_{q} a_{2}^{l^{\prime}}$. The interval $S_{p} S_{1}^{l} S_{k} U$ lies at a distance of at least $\rho^{l+2}$ from $A$ (since $S_{1}^{l+1} S_{k} U$ separates them), and $S_{q} S_{2}^{l^{\prime}} S_{j} U$ lies at a distance at least $\rho^{l^{\prime}+2}$ from $A$. Therefore, an upper bound for $|x-y|^{-\beta}$ for $x$ in the support of $\mu_{p, l, k}$ and $y$ in the support of $\mu_{q, l^{\prime}, j}$ is $\rho^{-\beta\left(2+\min \left(l, l^{\prime}\right)\right)}$. Altogether this produces an upper bound of

$$
\sum_{p, l} \sum_{q, l^{\prime}} a_{p} a_{q} a_{1}^{l} a_{2}^{l^{\prime}} \rho^{-\beta\left(2+\min \left(l, l^{\prime}\right)\right)} \leq c \sum_{l} \sum_{l^{\prime}} a_{1}^{l} a_{2}^{l^{\prime}} \rho^{-\beta \min \left(l, l^{\prime}\right)}
$$

for (3.26). Thus we need to show that

$$
\sum_{l \leq l^{\prime}} a_{1}^{l} a_{2}^{l^{\prime}} \rho^{-\beta l}
$$

is finite, and a similar expression with $a_{1}$ and $a_{2}$ interchanged.

Now recall that $\rho^{\beta}=\sum a_{j}^{2}$, so that (3.28) is equal to

$$
\sum_{l \leq l^{\prime}}\left(\frac{a_{1}}{\sum a_{j}^{2}}\right)^{l} a_{2}^{l^{\prime}} .
$$

If $a_{1}<\sum a_{j}^{2}$ then (3.29) is clearly finite. If $a_{1}=\sum a_{j}^{2}$ then (3.29) is equal to $\sum_{l^{\prime}} l^{\prime} a_{2}^{l^{\prime}}$, which is finite. If $a_{1}>\sum a_{j}^{2}$ then (3.29) is bounded by

$$
c \sum_{l^{\prime}}\left(\frac{a_{1} a_{2}}{\sum a_{j}^{2}}\right)^{l^{\prime}},
$$

which is finite since $a_{1} a_{2}<\sum a_{j}^{2}$. This completes the proof of (3.26).

Finally, we can replace the $L^{2}$ norms in (3.2) and (3.3) by $L^{4}$ norms, or more generally $L^{2 k}$ norms for $k$ a positive integer, in certain cases. If all the similarities $S_{j}$ have the same linear part, $S_{j} x=\rho R x+b_{j}$, then the distribution $f^{(k)}=f * f * \cdots * f \quad(k$ factors) is also self-similar. In fact if $f$ satisfies (2.1) then

$$
f^{(k)}=\sum_{j=1}^{m(k)} a_{j}^{(k)} f^{(k)} \circ\left(S_{j}^{(k)}\right)^{-1}
$$

with $S_{j}^{(k)} x=\rho R x+b_{j}^{(k)}$, where $a_{j}^{(k)}$ and $b_{j}^{(k)}$ satisfy $\left(\sum a_{j} e^{i b_{j} x}\right)^{k}=\sum a_{j}^{(k)} e^{i b_{j}^{(k)} x}$. If the similarities $S_{j}^{(k)}$ satisfy the strong open set condition (or the hypotheses of Lemma 3.8 in the case of measures) then Theorem 3.3 and Corollary 3.4 applied to $f^{(k)}$ yield

$$
\frac{1}{R^{n-\beta_{k}}} \int_{|x| \leq R}|F(x)|^{2 k} d x=\widetilde{H}_{k}(R)+o(1)
$$

as $R \rightarrow \infty$, where $\widetilde{H}_{k}(\rho R)=\widetilde{H}_{k}(R)$ and $\rho^{\beta_{k}}=\sum\left|a_{j}^{(k)}\right|^{2}$. 


\section{Fractal Plancherel formula}

In this section we consider a self-similar probability measure where the similarities are equicontractive and satisfy the strong open set condition and the weights are natural $($ all $=1 / m)$. The usual Cantor measure satisfies these hypotheses, but they are neither weaker nor stronger than the hypotheses for a generalized Cantor measure as defined in Part I. The requirement of natural weights implies that $\mu$ is locally uniformly $\beta$-dimensional $(\beta=-\log m / \log \rho$ in this case) and equal to the restriction of $\beta$-dimensional Hausdorff measure to the compact set $K$. In particular, we can apply the results of [S1] that show that for any $f \in L^{2}(d \mu)$,

$$
\frac{1}{R^{n-\beta}} \int_{|x| \leq R}\left|(f d \mu)^{\wedge}(x)\right|^{2} d x
$$

is bounded above and below (as $R \rightarrow \infty$ ) by multiples of $\int|f|^{2} d \mu$. In particular, this implies that $\widetilde{H}$ is bounded below. In fact we will show that

$$
\frac{1}{R^{n-\beta}} \int_{|x| \leq R}\left|(f d \mu)^{\wedge}(x)\right|^{2} d x=\left(\int|f|^{2} d \mu\right) \widetilde{H}(R)+E(R),
$$

where $E(R)$ satisfies (3.16). To do this we will construct a natural orthonormal basis of $L^{2}(d \mu)$ whose Fourier transforms are easily expressible in terms of $F=\hat{\mu}$.

Let $J^{\prime}=\left(j_{1}^{\prime}, \ldots, j_{N}^{\prime}\right)$ denote any multi-index of length $N$. Let $f_{J^{\prime}}$, denote the function which assumes the value $e^{2 \pi i J \cdot J^{\prime} / m}$ on $S_{J} K$ for $J$ of length $N$. We also allow $N=0$ and $J$ to be the empty set, with the corresponding function identically one on $K$. It is easy to see that the set of all such functions (for all values of $N$ ) is an orthonormal basis of $L^{2}(d \mu)$, and if we denote by $F_{J^{\prime}}$ the Fourier transform of $f_{J^{\prime}}$, then

$$
F_{J^{\prime}}(x)=\sum_{J \in \mathscr{J}_{N}} m^{-N} e^{2 \pi i J \cdot J^{\prime} / m} e^{i b_{J} \cdot x} F\left(\rho^{N} R_{J}^{*} x\right) .
$$

There is a vague resemblance to "wavelet" bases in that $F_{J^{\prime}}$ is manufactured out of dilates of $F$, but instead of translates we have multiplication by exponentials. Also, of course, the function $F$ is by no means localized.

Lemma 4.1. If $J^{\prime} \neq J^{\prime \prime}$ then

$$
\frac{1}{R^{n-\beta}} \int_{|x| \leq R} F_{J^{\prime}}(x) \overline{F_{J^{\prime \prime}}(x)} d x=E(R)
$$

while

$$
\frac{1}{R^{n-\beta}} \int_{|x| \leq R}\left|F_{J^{\prime}}(x)\right|^{2} d x=\widetilde{H}(R)+E(R),
$$

where $E(R)$ denotes a function satisfying (3.16).

Proof. We observe that

$$
\begin{aligned}
& \frac{1}{R^{n-\beta}} \int_{|x| \leq R}\left|F\left(\rho^{N} R_{J}^{*} x\right)\right|^{2} d x=\frac{\rho^{-N \beta}}{\left(\rho^{N} R\right)^{n-\beta}} \int_{|x| \leq \rho^{N} R}|F(x)|^{2} d x \\
& \quad=m^{N} H\left(\rho^{N} R\right)=m^{N} \widetilde{H}(R)+E(R)
\end{aligned}
$$


since $\rho^{\beta}=1 / m$. On the other hand, if $J^{\prime} \neq J^{\prime \prime}$ have the same length $N$ then

$$
\frac{1}{R^{n-\beta}} \int_{|x| \leq R} e^{i\left(b_{J^{\prime}}-b_{J^{\prime \prime}}\right) \cdot x} F\left(\rho^{N} R_{J^{\prime}}^{*} x\right) \overline{F\left(\rho^{N} R_{J^{\prime \prime}}^{*} x\right)} d x=E(R)
$$

by the proofs of Lemma 3.2 and Corollary 3.4. If we substitute the definition (4.2) into the right side of (4.4) and expand we see from (4.6) that the cross terms contribute $E(R)$, while each of the $m^{N}$ diagonal terms according to (4.5) contributes $m^{-2 N} m^{N} \widetilde{H}(R)+E(R)$, for a total of $\widetilde{H}(R)+E(R)$. This proves (4.4).

To prove (4.3) we first note that (4.2) remains valid if we take any $N$ greater than the length of $J^{\prime}$ (we interpret the dot product $J^{\prime} \cdot J$ to be the sum of $j_{k} j_{k}^{\prime}$ for $\left.k \leq \min \left(|J|,\left|J^{\prime}\right|\right)\right)$ because of (2.6). Thus, even if $J^{\prime}$ and $J^{\prime \prime}$ have different lengths, we can choose $N$ to be the greater of the two and use (4.2) for both $F_{J^{\prime}}$ and $F_{J^{\prime \prime}}$. If we substitute these into the left side of (4.3) and expand we again obtain $E(R)$ from the cross terms by (4.6), while the diagonal terms contribute

$$
m^{-N} \sum_{J \in \mathscr{J}_{N}} e^{2 \pi i J \cdot J^{\prime} / m} e^{-2 \pi i J \cdot J^{\prime \prime} / m} \widetilde{H}(R)+E(R)
$$

by (4.5). But the sum in (4.7) vanishes for $J^{\prime} \neq J^{\prime \prime}$ by a theorem of Lagrange, proving (4.3). Q.E.D.

Theorem 4.2. Let $\mu$ be a self-similar measure satisfying (2.1) for natural weights (all $a_{j}=1 / \mathrm{m}$ ) and similarities satisfying the equicontractive condition (all $\rho_{j}=$ $\rho)$ and the strong open set condition. Then (4.1) holds for any $f \in L^{2}(d \mu)$.

Proof. If $f$ is any finite linear combination of $f_{J^{\prime}}$ then (4.1) follows from (4.3) and (4.4). Then we obtain (4.1) for general $f \in L^{2}(d \mu)$ by a routine limiting argument because $\left\{f_{J^{\prime}}\right\}$ is an orthonormal basis and we have the a priori estimate

$$
\sup _{R} \frac{1}{R^{n-\beta}} \int_{|x| \leq R}\left|(f d \mu)^{\wedge}(x)\right|^{2} d x \leq c \int|f|^{2} d \mu
$$

(this is proved in [S1] under weaker hypotheses on $\mu$ ). Q.E.D.

\section{THE NONEQUiCONTRACTIVE CASE}

In this section we drop the equicontractive hypothesis, but retain the strong open set condition. The dimension $\beta$ can no longer be given by (3.1), but instead we determine $\beta$ by the condition

$$
\sum_{j=1}^{m} \rho_{j}^{-\beta}\left|a_{j}\right|^{2}=1
$$

It is easy to see that there is a unique real number $\beta$ satisfying (5.1) (the left side is increasing in $\beta$ and varies from zero as $\beta \rightarrow-\infty$ to $+\infty$ as $\beta \rightarrow+\infty)$ and (5.1) is consistent with (3.1) in the equicontractive case. We say that the $\left\{\rho_{j}\right\}$ are exponentially incommensurable if the ratios $\log \rho_{j} / \log \rho_{k}$ are all rational; or equivalently, if there exists $\rho$ such that

$$
\rho_{j}^{\nu_{j}}=\rho, \quad \nu_{j} \text { positive integers. }
$$


If not, we say that the $\left\{\boldsymbol{\rho}_{j}\right\}$ are exponentially commensurable.

We define the averages $h(t)$ and $H(R)$ by (3.3) and (3.2) with the new definition of $\beta$. If we substitute (2.5) into the definition of $h(t)$ and make a change of variable we see that

$$
\begin{aligned}
h(t) & =\sum_{j=1}^{m}\left|a_{j}\right|^{2} t^{(n-\beta) / 2} \int e^{-t|x|^{2}}\left|F\left(\rho_{j} R_{j}^{*} x\right)\right|^{2} d x+\sum_{j \neq k} a_{j} \bar{a}_{k} E_{j k}(t) \\
& =\sum_{j=1}^{m}\left|a_{j}\right|^{2} \rho_{j}^{-\beta} h\left(\rho_{j}^{-2} t\right)+\sum_{j \neq k} a_{j} \bar{a}_{k} E_{j k}(t)
\end{aligned}
$$

where

$$
E_{j k}(t)=t^{(n-\beta) / 2} \int e^{-t|x|^{2}} F\left(\rho_{j} R_{j}^{*} x\right) \overline{F\left(\rho_{k} R_{k}^{*} x\right)} d x .
$$

We can estimate $E_{j k}$ using (3.10) as before, and then use (5.3) to obtain both upper bounds and the asymptotic behavior of $h(t)$ as $t \rightarrow 0$.

Theorem 5.1. Let $f$ be a self-similar distribution of compact support, and suppose that $\left(\mathrm{A}_{0}\right)$ holds and the strong open set condition holds. Then $h(t)$ is uniformly bounded as $0<t \leq 1$. If the $\left\{\rho_{j}\right\}$ are exponentially incommensurable then $\lim _{t \rightarrow 0} h(t)$ exists. If the $\left\{\rho_{j}\right\}$ are exponentially commensurable then

$$
\lim _{t \rightarrow 0} h(t)-\tilde{h}(t)=0
$$

where

$$
\tilde{h}\left(\rho^{2} t\right)=\tilde{h}(t)
$$

and $\rho$ is given by (5.2).

Proof. We may assume that the $\left\{\rho_{j}\right\}$ are arranged in increasing order, so $\rho_{1} \leq$ $\rho_{j} \leq \rho_{m}$ for all $j$. Let $c_{N}=\sup \left\{h(t): \rho_{m}^{2 N} \rho_{1}^{2} \leq t \leq 1\right\}$. If $\rho_{m}^{2(N+1)} \rho_{1}^{2} \leq t \leq$ $\rho_{m}^{2 N} \rho_{1}^{2}$ then $\rho_{m}^{2 N} \rho_{1}^{2} \leq \rho_{j}^{-2} t \leq \rho_{m}^{2 N} \leq 1$ so

$$
c_{N+1} \leq c_{N}+\sum_{j \neq k} \sup \left\{\left|E_{j k}(t)\right|: \rho_{m}^{2(N+1)} \rho_{1}^{2} \leq t \leq \rho_{m}^{2 N} \rho_{1}^{2}\right\}
$$

by (5.3) and (5.1). In view of (3.10) this proves the boundedness of $h(t)$ on $(0,1]$. By the same reasoning as in the proof of Lemma 3.2 and Theorem 3.3 we have (5.5) holding, where $\tilde{h}(t)$ is bounded and satisfies

$$
\tilde{h}(t)=\sum_{j=1}^{m}\left|a_{j}\right|^{2} \rho_{j}^{-\beta} \tilde{h}\left(\rho_{j}^{-2} t\right) .
$$

If we write $\varphi(s)=\tilde{h}\left(e^{s}\right)$ then (5.7) becomes

$$
\varphi(s)=\sum_{j=1}^{m} \lambda_{j} \varphi\left(s+\gamma_{j}\right),
$$

where $\sum \lambda_{j}=1,0<\lambda_{j}<1$, and $\gamma_{j}=-2 \log \rho_{j}>0$. Taking the Fourier transform of $(5.8)$ yields

$$
\left(1-\sum_{j=1}^{m} \lambda_{j} e^{i \gamma_{j} \xi}\right) \hat{\varphi}(\xi)=0 .
$$


If the $\left\{\rho_{j}\right\}$ are exponentially incommensurable then $\left(1-\sum \lambda_{j} e^{i \gamma_{j} \xi}\right)$ vanishes only at zero, where it has a simple zero, so the only solutions of (5.8) are constant. Thus $\tilde{h}$ is constant in this case. If, on the contrary, (5.2) holds, then $1-\lambda_{j} e^{i \gamma_{j} \xi}$ is periodic and has simple zeros at multiples of $\pi / \log \rho$, which implies the periodicity (5.6). Q.E.D.

Theorem 5.2. Let $\left\{a_{j}\right\}$ be positive weights satisfying $\left(\mathrm{A}_{0}\right)$, so $f=\mu$ is a selfsimilar measure. Then $h(t)$ is bounded below by a positive constant on $0<t \leq$ 1 .

Proof. We will use an iteration of (2.5) that is more controlled than (2.6). We will obtain

$$
F(x)=\sum_{J \in \mathscr{A}} a_{J} e^{i b_{J} \cdot x} F\left(\rho_{J} R_{J}^{*} x\right)
$$

for certain finite sets $\mathscr{A}$ of multi-indices (of varying length) with the property that all $\rho_{J}$ for $J \in \mathscr{A}$ are of comparable size. Now it is clear that we can generate many identities of the form (5.9) by a recursive procedure. If we have (5.9) for a fixed $\mathscr{A}$, just choose one $J \in \mathscr{A}$ and replace $F\left(\rho_{J} R_{J}^{*} x\right)$ by

$$
\sum_{j=1}^{m} a_{j} e^{i b_{j} \cdot \rho_{J} R_{j}^{*} x} F\left(\rho_{j} \rho_{J} R_{j}^{*} R_{J}^{*} x\right),
$$

which is equal to it by (2.5). Now the substitution of (5.10) in (5.9) yields another identity of the form (5.9) with $\mathscr{A}$ replaced by another set $\mathscr{A}^{\prime}$ obtained from $\mathscr{A}$ by removing $J$ and adjoining $(J, 1), \ldots,(J, m)$. We can always get started since (2.5) is of the form (5.9) with $\mathscr{A}=\{1, \ldots, m\}$. If we set a target value $\lambda$ (chosen small enough, say $\lambda<\rho_{1}$ ) and we perform the above the procedure on $J$ if and only if $\rho_{J}>\lambda$, then after a finite number of steps we will arrive at a set we denote $\mathscr{A}(\lambda)$ for which (5.9) holds with

$$
\rho_{1} \lambda \leq \rho_{J} \leq \lambda \text { for all } J \in \mathscr{A}(\lambda) \text {. }
$$

Suppose for a moment that $\sum_{J \in \mathscr{A}(\lambda)} a_{J} e^{i b_{J} \cdot x}$ were a good approximation for $F(x)$ in computing $h(t)$. A simple computation shows

$$
\begin{aligned}
t^{(n-\beta) / 2} & \int e^{-t|x|^{2}}\left|\sum_{J \in \mathscr{A}(\lambda)} a_{J} e^{i b_{J} \cdot x}\right|^{2} d x \\
= & \pi^{n / 2} t^{-\beta / 2} \sum_{J \in \mathscr{A}(\lambda)} \sum_{J^{\prime} \in \mathscr{A}(\lambda)} a_{J} a_{J^{\prime}} e^{-\left|b_{J}-b_{J^{\prime}}\right|^{2} / 4 t}
\end{aligned}
$$

and since all terms are postive we obtain a lower bound of

$$
\pi^{n / 2} t^{-\beta / 2} \sum_{J \in \mathscr{A}(\lambda)} a_{J}^{2}
$$

just by selecting the diagonal terms in the sum. If we choose $\lambda=t^{1 / 2}$ then $t^{-\beta / 2} \geq \rho_{1}^{\beta} \rho_{J}^{-\beta}$ by (5.11) so $\pi^{n / 2} \rho_{1}^{\beta} \sum_{J \in \mathscr{A}(\lambda)} \rho_{J}^{-\beta} a_{J}^{2}$ is a lower bound for (5.12). But from (5.1) and the way $\mathscr{A}(\lambda)$ is constructed we see that

$$
\sum_{J \in \mathscr{A}(\lambda)} \rho_{J}^{-\beta} a_{J}^{2}=1
$$


and so

$$
t^{(n-\beta) / 2} \int e^{-t|x|^{2}}\left|\sum_{J \in \mathscr{A}(\sqrt{t})} a_{J} e^{i b_{J} \cdot x}\right|^{2} d x \geq \pi^{n / 2} \rho_{1}^{\beta} .
$$

This is the right kind of lower bound, but unfortunately (5.9) does not allow us to immediately apply $(5.14)$ to estimate $h(t)$. What we do know by Theorem 2.3 is that

$$
F_{N}(x)=\sum_{J \in \mathscr{F}_{N}} a_{J} e^{i b_{J} \cdot x}
$$

converges to $F(x)$ uniformly on compact sets, and $F_{N}$ is uniformly bounded by one, so that for any fixed $t$ we can choose $N$ large enough that

$$
\begin{aligned}
t^{(n-\beta) / 2} \int e^{-t|x|^{2}}|F(x)|^{2} d x & \geq \frac{1}{2} t^{(n-\beta) / 2} \int e^{-t|x|^{2}}\left|F_{N}(x)\right|^{2} d x \\
& =\frac{1}{2} \pi^{n / 2} t^{-\beta / 2} \sum_{J \in \mathscr{J}_{N}} \sum_{J^{\prime} \in \mathscr{J}_{N}} a_{J} a_{J^{\prime}} e^{-\left|b_{J}-b_{J^{\prime}}\right|^{2} / 4 t}
\end{aligned}
$$

Without loss of generality we may take $N$ large enough that $\mathscr{A}(\sqrt{t}) \subseteq \mathscr{J}_{N}$. However, unlike the case of (5.12), it would be disastrous in (5.15) to throw away all the nondiagonal terms. Instead, we retain just those terms corresponding to $J$ and $J^{\prime}$ that both contain the same initial segment in $\mathscr{A}(\sqrt{t})$.

Changing notation slightly, write $J$ for a fixed multi-index in $\mathscr{A}(\sqrt{t})$, of length $|J| \leq N$, and write $J J^{\prime}$ and $J J^{\prime \prime}$ for multi-indices in $\mathscr{J}_{N}$ whose first $|J|$ entries agree with $J$ (so $J^{\prime}$ and $J^{\prime \prime}$ are in $\mathscr{J}_{N-|J|}$ ). Then the lower bound we extract from $(5.15)$ is

$$
h(t) \geq \frac{1}{2} \pi^{n / 2} t^{-\beta / 2} \sum_{J \in \mathscr{A}(\sqrt{t})} \sum_{J^{\prime} \in \mathcal{I}_{N-|J|}} \sum_{J^{\prime \prime} \in \mathcal{I}_{N-|J|}} a_{J}^{2} a_{J^{\prime}} a_{J^{\prime \prime}} e^{-\left|b_{J J^{\prime}}-b_{J J^{\prime \prime}}\right|^{2} / 4 t}
$$

Now we need an upper bound for $\left|b_{J J^{\prime}}-b_{J J^{\prime \prime}}\right|$. But we have the explicit formula (with $|J|=k$ )

$$
\begin{aligned}
b_{J J^{\prime}}= & b_{j_{1}}+\rho_{j_{1}} R_{j_{1}} b_{j_{2}}+\cdots+\left(\rho_{j_{1}} \cdots \rho_{j_{k-1}}\right)\left(R_{j_{1}} \cdots R_{j_{k-1}}\right) b_{j_{k}} \\
& +\rho_{J} R_{J}\left(b_{j_{k+1}^{\prime}}+\rho_{j_{k+1}^{\prime}} R_{j_{k+1}^{\prime}} b_{j_{k+2}^{\prime}}+\cdots\right. \\
& \left.+\left(\rho_{j_{k+1}^{\prime}} \cdots \rho_{j_{N-1}^{\prime}}\right)\left(R_{j_{k+1}^{\prime}} \cdots R_{j_{N-1}^{\prime}}\right) b_{j_{N}^{\prime}}\right)
\end{aligned}
$$

and a similar expression for $b_{J J^{\prime \prime}}$, so $b_{J J^{\prime}}-b_{J J^{\prime \prime}}=\rho_{J} R_{J} \tilde{b}$, where

$$
\begin{aligned}
\tilde{b}= & \left(b_{j_{k+1}^{\prime}}-b_{j_{k+1}^{\prime \prime}}\right)+\cdots+\left(\rho_{j_{k+1}^{\prime}} \cdots \rho_{j_{N-1}^{\prime}}\right)\left(R_{j_{k+1}^{\prime}} \cdots R_{j_{N-1}^{\prime}}\right) b_{j_{N}^{\prime}} \\
& -\left(\rho_{j_{k+1}^{\prime \prime}} \cdots \rho_{j_{N-1}^{\prime \prime}}\right)\left(R_{j_{k+1}^{\prime \prime}} \cdots R_{j_{N-1}^{\prime \prime}}\right) b_{j_{N}^{\prime \prime}} .
\end{aligned}
$$

Clearly

$$
|\tilde{b}| \leq 2 M\left(1+\rho_{m}+\rho_{m}^{2}+\cdots+\rho_{m}^{N-k-2}\right) \leq \frac{2 M}{1-\rho_{m}},
$$

where $M=\max _{1 \leq j \leq m}\left|b_{j}\right|$, so

$$
\left|b_{J J^{\prime}}-b_{J J^{\prime \prime}}\right| \leq \frac{2 M}{1-\rho_{m}} \rho_{J} \leq \frac{2 M t^{1 / 2}}{1-\rho_{m}}
$$


by (5.11). Thus

$$
\frac{\left|b_{J J^{\prime}}-b_{J J^{\prime \prime}}\right|^{2}}{4 t} \leq \frac{M^{2}}{\left(1-\rho_{m}\right)^{2}}
$$

and substituting this in (5.16) we obtain

$$
h(t) \geq \frac{1}{2} \pi^{n / 2} e^{-M^{2} /\left(1-\rho_{m}\right)^{2}} \sum_{J \in \mathscr{A}(\sqrt{t})} t^{-\beta / 2} a_{J}^{2} \sum_{J^{\prime} \in \mathscr{f}_{N-|J|}} a_{J^{\prime}} \sum_{J^{\prime \prime} \in \mathscr{f}_{N-|J|}} a_{J^{\prime \prime}} .
$$

But $\sum_{J^{\prime}} a_{J^{\prime}}=\sum_{J^{\prime \prime}} a_{J^{\prime \prime}}=1$ and so

$$
h(t) \geq \frac{1}{2} \pi^{n / 2} e^{-M^{2} /\left(1-\rho_{m}\right)^{2}} \rho_{1}^{\beta}
$$

by (5.11) and (5.13) as before. Q.E.D.

Corollary 5.3. Let $f=\mu$ be a self-similar measure with positive weights satisfying $\left(\mathrm{A}_{0}\right)$, and assume the strong open set condition. Then $H(R)=\widetilde{H}(R)+E(R)$ with

$$
\lim _{R \rightarrow \infty} \int_{R \rho}^{R} E(r) \frac{d r}{r}=0,
$$

where $\widetilde{H}(R)$ is a bounded function bounded away from zero satisfying

$$
\widetilde{H}(\rho R)=\widetilde{H}(R)
$$

if the $\left\{\rho_{j}\right\}$ are exponentially commensurable ( $\rho$ given by (5.2)), or

$$
\lim _{R \rightarrow \infty} H(R)=\widetilde{H}
$$

a positive constant if the $\left\{\rho_{j}\right\}$ are exponentially incommensurable.

Proof. This follows as before by combining Theorems 5.1 and 5.2 with a Tauberian argument. Q.E.D.

Note that we do not claim that the constant $\widetilde{H}$ in (5.19) is any sort of universal constant depending only on the dimension $\beta$. It may depend on $\mu$ in an unspecified manner.

Recall from Part I and $[\mathrm{H}]$ that there are natural weights associated to the set of similarities $\left\{S_{j}\right\}$ defined by

$$
a_{j}=\rho_{j}^{s},
$$

where $s$ is the unique positive number for which $\left(\mathrm{A}_{0}\right)$ holds. Then, under the open set condition, $s=\beta$ and the measure $\mu$ is the restriction of $\beta$ dimensional Hausdorff measure to $K$.

Corollary 5.4. Let $\mu$ be a self-similar measure associated to a set of similarities satisfying the strong open set condition with natural weights (5.20). Then (4.1) holds for any $f \in L^{2}(d \mu)$ with $\widetilde{H}(R)$ as in Corollary 5.3. In particular, if $\left\{\rho_{j}\right\}$ are exponentially incommensurable, then

$$
\lim _{R \rightarrow \infty} \frac{1}{R^{n-\beta}} \int_{|x| \leq R}\left|(f d \mu)^{\wedge}(x)\right|^{2} d x=\widetilde{H} \int|f|^{2} d \mu .
$$


Proof. The proof follows the argument in Lemma 4.1 and Theorem 4.2, with the modification that $f_{J^{\prime}}$ is defined to assume the value $m^{-N / 2} a_{J}^{-1 / 2} e^{2 \pi i J \cdot J^{\prime} / m}$ on $S_{J} K$, and so

$$
F_{J^{\prime}}(x)=\sum_{J \in \mathscr{J}_{N}} m^{-N / 2} a_{J}^{1 / 2} e^{2 \pi i J \cdot J^{\prime} / m} e^{i b_{J} \cdot x} F\left(\rho_{J} R_{J}^{*} x\right) \text {. Q.E.D. }
$$

\section{Generalizations}

In this section we discuss briefly a number of different generalizations of the previous results.

(i) Self-affine distributions. Instead of requiring contractive similarities $S_{j}$ in the definition (2.1), we could require merely contractive affine transformations $S_{j} x=A_{j} x+b_{j}$, where $A_{j}$ is a contractive linear transformation. In place of (2.5) we would have

$$
F(x)=\sum_{j=1}^{m} a_{j} e^{i b_{j} \cdot x} F\left(A_{j}^{*} x\right) .
$$

The results of $\S 2$ extend easily with the obvious modifications. However, none of the results in $\S \S 3-5$ seem to extend to this case.

(ii) Infinite sums. In place of the finite sum in (2.1) we could allow an infinite sum, as long as we require $\sum_{j=1}^{\infty}\left|a_{j}\right|<\infty$ and $\lim _{j \rightarrow \infty} \rho_{j}=0$. Again the results of $\S 2$ extend to this case. Under certain additional assumptions the results of $\S 5$ extend as well. What is needed is a quantitative version of the strong open set condition. Let $d_{j k}$ denote the distance between $S_{j} U$ and $S_{k} U$, which is positive for $j \neq k$ by the strong open set condition. For simplicity we deal only with the case of positive weights. We claim that the condition

$$
\sum_{j \neq k} a_{j} a_{k} d_{j k}^{-\beta}<\infty
$$

is sufficient for the extension of Theorem 5.1 to hold. The problem is to control the infinite sum $\sum_{j \neq k} a_{j} a_{k} E_{j k}(t)$, as in the proof of Lemma 3.2, which requires the convergence of the series

$$
\sum_{q=1}^{\infty} \sum_{j \neq k} a_{j} a_{k} E_{j k}\left(\rho^{2 q} t\right),
$$

which can be estimated by

$$
c t^{-\beta / 2} \sum_{q=1}^{\infty} \sum_{j \neq k} a_{j} a_{k} \rho^{-\beta q} e^{-d_{j k}^{2} / 4 t \rho^{2 q}} .
$$

But now an easy estimate shows

$$
t^{-\beta / 2} \sum_{q=1}^{\infty} \rho^{-\beta q} e^{-d_{j k}^{2} / 4 t \rho^{2 q}} \leq c d_{j k}^{-\beta}
$$

and so (6.1) implies that (6.2) is finite.

Theorem 5.2 also extends to this case under certain additional hypotheses. To see this we observe that the construction of $\mathscr{A}(\lambda)$ can be carried out as 
before, but now only the second inequality in (5.11) will hold. We still have the estimate (5.16) and the proof leads to the lower bound

$$
h(t) \geq c \sum_{J \in \mathscr{A}(\sqrt{t})} t^{-\beta / 2} a_{J}^{2}=c \sum_{J \in \mathscr{A}(\sqrt{t})}\left(\rho_{J}^{2} / t\right)^{\beta / 2} \rho_{J}^{-\beta} a_{J}^{2} .
$$

If we had a lower bound for $\rho_{J}^{2} / t$ we would be done, but this is no longer true over all of $\mathscr{A}(\sqrt{t})$. Instead, we need to show that it is true for a substantial portion of $\mathscr{A}(\sqrt{t})$. Define the subset $\mathscr{A}_{1}(\sqrt{t})$ by selecting those $J$ for which $\varepsilon \sqrt{t} \leq \rho_{J}$ (we also have $\rho_{J} \leq \sqrt{t}$ by the construction of $\mathscr{A}(\sqrt{t})$ ), where $\varepsilon$ is a fixed parameter satisfying $0<\varepsilon<1$. Our lower bound is then

$$
h(t) \geq c \sum_{J \in \mathscr{A}_{1}(\sqrt{t})} \rho_{J}^{-\beta} a_{J}^{2}
$$

(of course $c$ depends on $\varepsilon$ ). In view of (5.13) we need an estimate of the form

$$
\sum_{J \in \mathscr{A}_{1}(\sqrt{t})} \rho_{J}^{-\beta} a_{J}^{2} \geq c_{1} \sum_{J \in \mathscr{A}(\sqrt{t})} \rho_{J}^{-\beta} a_{J}^{2}
$$

with $c_{1}>0$ independent of $t$.

The kind of hypothesis that will guarantee this involves the function

$$
q(\lambda)=\sum_{\rho_{j \leq i}} a_{j}^{2} \rho_{j}^{-\beta}
$$

Suppose we have

$$
q(\varepsilon \lambda) \leq \delta q(\lambda)
$$

for some $\delta<1$. This would imply

$$
\sum_{\varepsilon \lambda<\rho_{j} \leq \lambda} a_{j}^{2} \rho_{j}^{-\beta}=q(\lambda)-q(\varepsilon \lambda) \geq\left(\delta^{-1}-1\right) q(\varepsilon \lambda)
$$

and $\delta^{-1}-1>0$. Now if $J$ is in $\mathscr{A}(\sqrt{t})$ but not in $\mathscr{A}_{1}(\sqrt{t})$, we must have $\rho_{j_{1}} \cdots \rho_{j_{N-1}}>\sqrt{t}$, but $\rho_{j_{1}} \cdots \rho_{j_{N}} \leq \varepsilon \sqrt{t}$. Fix $j_{1}, \ldots, j_{N-1}$ and vary $j_{N}=j$. Some of the resulting $J$ will belong to $\mathscr{A}_{1}(\sqrt{t})$, some will belong to $\mathscr{A}(\sqrt{t})$ but not $\mathscr{A}_{1}(\sqrt{t})$, and some will not belong to $\mathscr{A}(\sqrt{t})$. If we abbreviate $\rho_{j_{1}} \cdots \rho_{j_{N-1}}=A$ then the conditions under which these three cases occur are

$$
\begin{array}{ll}
\varepsilon \sqrt{t}<A \rho_{j} \leq \sqrt{t} & \text { for } J \in \mathscr{A}_{1}(\sqrt{t}), \\
A \rho_{j} \leq \varepsilon \sqrt{t} & \text { for } J \in \mathscr{A}_{1}(\sqrt{t}) \backslash \mathscr{A}(\sqrt{t}), \\
\sqrt{t}<A \rho_{j} & \text { for } J \notin \mathscr{A}(\sqrt{t}) .
\end{array}
$$

Condition (6.8) with $\lambda=A^{-1} \sqrt{t}$ says that $\sum a_{j}^{2} \rho_{j}^{-\beta}$ in the first case is at least $\left(\delta^{-1}-1\right)$ times the same sum in the second case, and this is the same as for $\sum a_{J}^{2} \rho_{J}^{-\beta}$ because all terms have the common factor $a_{j_{1}}^{2} \cdots a_{j_{N-1}}^{2} \rho_{j_{1}}^{-\beta} \cdots \rho_{j_{N-1}}^{-\beta}$, which is being held fixed. This gives the estimate (6.5) with $c_{1}=\delta^{-1}-1$. Thus hypothesis $(6.7)$ is sufficient for Theorem 5.2 to hold in this case. Similarly Corollaries 5.3 and 5.4 hold under the additional assumptions (6.1) and (6.7).

(iii) Noncompact support. If we drop the requirement that the distribution have compact support then we obtain many more self-similar distributions. For 
example, if $\mu$ denotes the usual Cantor measure on [0,1], we can also define a grand Cantor measure $\tilde{\mu}$ on $[0, \infty)$ which is the sum of $\mu$ translated by all nonnegative integers expressible base 3 without using the digit 1 . It may be argued that $\tilde{\mu}$ is even more self-similar than $\mu$ since it looks the same at all scales, large or small. It is easy to see that $\tilde{\mu}$ satisfies the self-similar identity

$$
\tilde{\mu}=\frac{1}{2} \tilde{\mu} \circ S^{-1}
$$

for $S x=\frac{1}{3} x$. This is of the form (2.1) but none of the conditions $\left(\mathrm{A}_{k}\right)$ is satisfied, so there are no compactly supported solutions of (6.9). However, $\tilde{\mu}$ is by no means the only solution of (6.9). In fact any dilate of $\tilde{\mu}$ also satisfies (6.9), which already shows that the space of solutions is infinite dimensional. But also the measure $|x|^{-\alpha} d x$ satisfies (6.9) for $\alpha=1-\log 2 / \log 3$.

The following is a general procedure for constructing infinite-dimensional spaces of solutions of (2.1) out of a single compactly supported solution $f$ in the equicontractive case: Let $G$ be any bounded radial function satisfying the periodicity condition

$$
G(\rho x)=G(x) .
$$

Then $G=\hat{g}$ for a tempered distribution $g$, and it is easy to see that $g * f$ (whose Fourier transform is $F(x) G(x)$ ) also satisfies (2.1).

Even in the nonequicontractive case we can give a general construction under certain restrictions. The main assumption is $\rho_{1}<\rho_{2}$ (with the $\left\{\rho_{j}\right\}$ arranged in increasing order) so that we can compose (2.1) with $S_{1}$ and solve to obtain

$$
\left\{\begin{array}{l}
f=\sum_{j=1}^{m} \tilde{a}_{j} f \circ \tilde{S}_{j}^{-1} \quad \text { with } \\
\tilde{a}_{1}=a_{1}^{-1}, \tilde{a}_{j}=a_{1}^{-1} a_{j}, \quad j \geq 2, \\
\tilde{S}_{1}=S_{1}^{-1}, \tilde{S}_{j}=S_{1}^{-1} \circ S_{j}, \quad j \geq 2,
\end{array}\right.
$$

and now the $\tilde{S}_{j}$ are all expansive similarities. It is easy to show from (6.11) that all solutions must be tempered distributions. Thus we can work with the Fourier transform and (2.5). To construct solutions of (2.5) we define $F$ arbitrarily on the annular region $A_{0}=\left\{\rho_{1}<|x| \leq 1\right\}$, say bounded by one. Then use (2.5) inductively to extend the definition to the regions $A_{k}=\left\{\rho_{m}^{-k+1}<|x| \leq \rho_{m}^{-k}\right\}$ for $k=1,2, \ldots$. We have the estimate

$$
|F(x)| \leq\left(\sum_{j=1}^{m}\left|a_{j}\right|\right)^{k}
$$

on $A_{k}$ which is a polynomial growth rate at infinity. We use

$$
F\left(\rho_{1} R_{1}^{*} x\right)=a_{1}^{-1} e^{-i b_{1} \cdot x}\left(F(x)-\sum_{j=2}^{m} a_{j} e^{i b_{j} \cdot x} F\left(\rho_{j} R_{j}^{*} x\right)\right)
$$

to inductively extend $F$ to the regions

$$
A_{-k}=\left\{\rho_{1}^{k+1} \rho_{2}^{-k} \leq|x| \leq \rho_{1}^{k} \rho_{2}^{-k+1}\right\}
$$

for $k=1,2, \ldots$ with the estimate

$$
|F(x)| \leq\left|a_{1}\right|^{-k}\left(1+\sum_{j=2}^{m}\left|a_{j}\right|\right)^{k}
$$


on $A_{k}$. This implies $|F(x)| \leq c|x|^{-\alpha}$ for $|x| \leq 1$ with

$$
\alpha=\frac{\log \left(\left|a_{1}\right|^{-1}\left(1+\sum_{j=2}^{m}\left|a_{j}\right|\right)\right)}{\log \rho_{2} / \rho_{1}} .
$$

Therefore, if we assume

$$
\log \left(\left|a_{1}\right|^{-1}\left(1+\sum_{j=2}^{m}\left|a_{j}\right|\right)\right)<n \log \rho_{2} / \rho_{1},
$$

this procedure yields locally integrable solutions of $(2.5)$, hence tempered distributions satisfying (2.1), and clearly the space of solutions is infinite dimensional. of

(iv) $L^{p}$ means. In place of (1.1) we could consider the asymptotic behavior

$$
H_{p}(R)=\frac{1}{R^{n-\beta_{p}}} \int_{|x| \leq R}|\hat{\mu}(x)|^{p} d x
$$

for appropriate values of $\beta_{p}$. At the end of $\S 3$ we indicated how to obtain some results for $p$ equal to an even integer. However, the results of numerical experiments carried out by Maria Korolov suggest that the asymptotic periodicity results for $H(R)$ should remain true for $H_{p}(R)$ for all $p$ satisfying $0<p<\infty$. The possibility of interpolating between established values of $p$ comes as no surprise (of course the usual interpolation arguments do not give the desired results), but the extension below $p=1$ seems rather amazing. Certainly it will require entirely new techniques to prove such results.

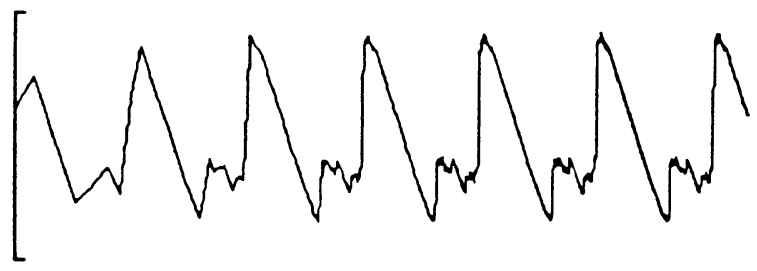

Figure 2. $p=3, \beta=.792$

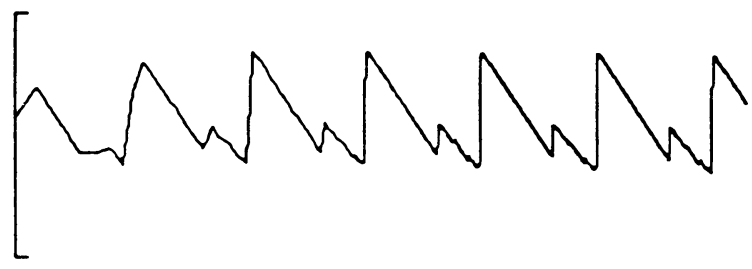

FIGURE 3. $p=5.5 \quad \beta=.965$

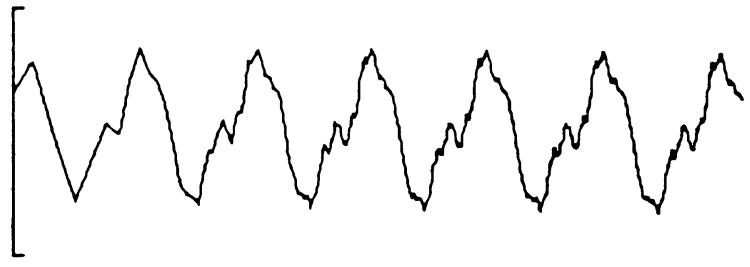

Figure 4. $p=.5 \beta=.230$ 
In Figures 2, 3, and 4, we show the graph of $H_{p}(R)$ on a logarithmic $R$-scale for different values of $p$ and $\beta_{p}$ (determined experimentally).

\section{REFERENCES}

[F] W. Feller, An introduction to probability theory and its applications, Vol. 2, 2nd ed., Wiley, New York, 1971.

[HL] S. Hudson and M. Leckband, Hardy's inequality and fractal measures, J. Funct. Anal. 108 (1992), 133-160.

[H] J. E. Hutchinson, Fractals and self-similarity, Indiana Univ. Math. J. 30 (1981), 713-747.

[Ly] S. Lalley, The packing and covering functions of some self-similar fractals, Indiana Univ. Math. J. 37 (1988), 699-709.

[Lu] Ka-Sing Lau, Fractal measures and mean p-variations, J. Funct. Anal. 108 (1992), 427-457.

[S1] R. S. Strichartz, Fourier asymptotics of fractal measures, J. Funct. Anal. 89 (1990), 154-187.

[S2] _ Besicovitch meets Wiener-Fourier expansions and fractal measures, Bull. Amer. Math. Soc. (N.S.) 20 (1989), 55-59.

[S3] _, Self-similar measures and their Fourier transforms. I, Indiana Univ. Math. J. 39 (1990), 797-817.

[S4] _ Spectral asymptotics of fractal measures on Riemannian manifolds, J. Funct. Anal. 102 (1991), 176-205.

[T] M. E. Taylor, Pseudodifferential operators, Princeton Univ. Press, Princeton, N.J., 1981.

[W] N. Wiener, The Fourier integral and certain of its applications, Dover, New York, 1933.

\section{ADDED IN PROOF}

[DL] I. Daubechies and J. Lagarias, Two-scale difference equations. I and II, SIAM J. Math. Anal. 22 (1991), 1388-1410, 23 (1992), 1031-1079.

[Lu2] Ka-Sing Lau, Dimension of a family of singular Bernoulli convolutions, preprint.

[LW] K.-S. Lau and J. Wang, Mean quadratic variations and Fourier asymptotics of self-similar mesures, Monatsh. Math. (to appear).

[JRS] P. Janardhan, D. Rosenblum and R. S. Strichartz, Numerical experiments in Fourier asymptotics of Cantor measures and weavelets, Experimental Math. (to appear).

[S5] R. S. Strichartz, Wavelet expansions of fractal measures, J. Geom. Anal. 1 (1991), 269-289.

[S6] _ Self-similar measures and their Fourier transforms. III, preprint.

[S7] _ Self-similarity on nilpotent Lie groups, Contemp. Math. (to appear).

Department of Mathematics, White Hall, Cornell University, Ithaca, New York 14853 